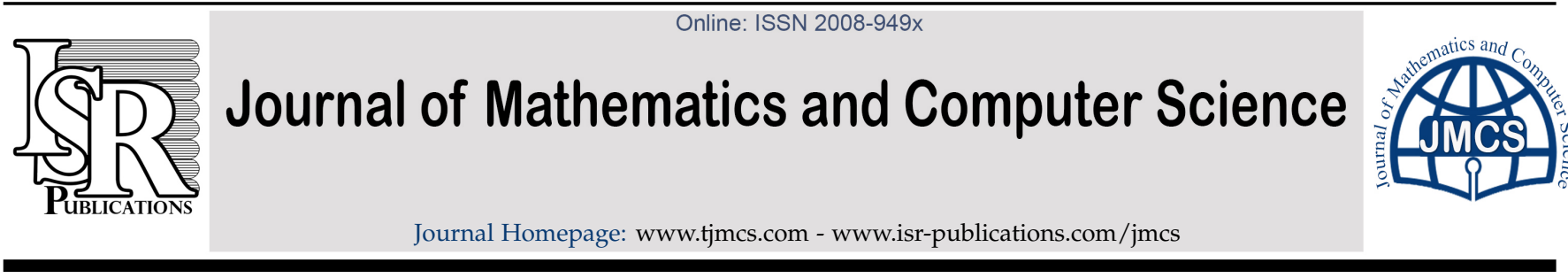

\title{
A sufficient condition for coinciding the Green graphs of semigroups
}

\author{
Mohammad Reza Sorouhesh ${ }^{\mathrm{a}, *}$, Hossein Doostie $^{\mathrm{a}}$, Colin M. Campbell \\ ${ }^{a}$ Department of Mathematics, Tehran Science and Research Branch Islamic Azad University, Tehran, 14515/1775, Iran. \\ ${ }^{b}$ School of Mathematics and Statistics, University of St. Andrews, North Haugh, St. Andrews, Fife, KY16 9SS Scotland, UK.
}

\begin{abstract}
A necessary condition for coinciding the Green graphs $\Gamma_{\mathcal{L}}(S), \Gamma_{\mathcal{R}}(S), \Gamma_{\mathcal{J}}(S), \Gamma_{\mathcal{D}}(S)$ and $\Gamma_{\mathcal{H}}(S)$ of a finite semigroup $S$ has been studied by Gharibkhajeh [A. Gharibkhajeh, H. Dosstie, Bull. Iranian Math. Soc., 40 (2014), 413-421]. Gharibkhajeh et al. proved that the coinciding of Green graphs of a finite semigroup S implies the regularity of S. However, the converse is not true because of certain well-known examples of finite regular semigroups. We look for a sufficient condition on non-group semigroups that implies the coinciding of the Green graphs. Indeed, in this paper we prove that for every non-group quasi-commutative finite semigroup, all of the Green graphs are isomorphic. (C)2017 all rights reserved.
\end{abstract}

Keywords: Quasi-commutativity, finitely presented semigroups, Green relations, Green graphs. 2010 MSC: 20M05, 05C99.

\section{Introduction}

Let $S$ be a finite semigroup. Following the notation of [4], the left Green Graph $\Gamma_{\mathcal{L}}(S)$ is an undirected graph with vertices $\mathcal{L}_{i},(1 \leqslant i \leqslant t)$ where the $\mathcal{L}_{i}$ are the left Green classes of the semigroup $S$ and two vertices $\mathcal{L}_{\mathfrak{i}}, \mathcal{L}_{j}$ are adjacent in $\Gamma_{\mathcal{L}}(S)$ if and only if $\operatorname{gcd}\left(\left|\mathcal{L}_{i}\right|,\left|\mathcal{L}_{j}\right|\right)>1$. These graphs are indeed the generalization of the conjugacy graphs of finite groups studied by Adan-Bante [1]. The right Green graph $\Gamma_{\mathcal{R}}(S)$, the intersection Green graph $\Gamma_{\mathcal{H}}(S)$, the join Green graph $\Gamma_{\mathcal{D}}(S)$, and finally the $\mathcal{J}$-classes Green graph $\Gamma_{\mathcal{J}}(S)$ are defined in a similar way. Investigating these graphs is of interest because of their ability in identifying certain types of finite semigroups, the non-group non-regular quasi-commutative semigroups. As usual, an associative algebraic structure $(S, \cdot)$ is called quasi-commutative if, for every elements $a, b \in S$, there exists a positive integer $r$ such that $a b=b^{r} a$. For useful information on quasi-commutative semigroups and examples, one may see [2,3,5-7]. Our main results on this type of semigroup are the following:

Proposition A. For every non-commutative quasi-commutative semigroup S, all Green graphs are isomorphic.

Proposition B. If $\mathrm{b}$ is a non idempotent element of a nowhere commutative quasi-commutative finite semigroup $\mathrm{S}$, then $\mathrm{b}$ is regular if and only if $\left|[\mathrm{b}]_{\mathrm{g}}\right|>1$.

\footnotetext{
${ }^{*}$ Corresponding author

Email addresses: sorouhesh@azad.ac.ir (Mohammad Reza Sorouhesh), doostih@gmail.com (Hossein Doostie), cmc@st-andrews .ac.uk (Colin M. Campbell)
}

doi:10.22436/jmcs.017.02.03 
Proposition C. Let $\mathrm{S}$ be a finite non-regular nowhere commutative quasi-commutative semigroup. Then all of the Green graphs of $\mathrm{S}$ are isomorphic to $\mathrm{nK}_{1} \cup \mathrm{K}_{\mathrm{m}}$ where $\mathrm{m}$ is the number of $\mathcal{L}$-classes and $\mathrm{n}$ is the number of non-regular non idempotent elements of S. Moreover, these graphs are not complete.

\section{The proofs}

We start the proofs with a key lemma.

Lemma 2.1. Every non idempotent regular element $\mathrm{b}$ of a finite semigroup $\mathrm{S}$ satisfies $\left|[\mathrm{b}]_{\mathfrak{J}}\right|>1$.

Proof. For a regular element $b \in S$, we may use a method of proof similar to the proof of Lemma 1.14. of [3]. Indeed, there exists an element $x \in S$ such that $b=b x b$, and then $y=x b x$ is an inverse for $b$. This yields $y b y=(x b x) b(x b x)=x(b x b)(x b x)=x(b x b) x=x b x=y$ and $b y b=b(x b x) b=(b x b) x b=b x b=b$. Let $y \neq b$. So, $y b y=y$ and $b y b=b$ implies that $y \in[b]_{\mathcal{J}}$ and therefore $\left|[b]_{\mathcal{J}}\right|>1$. If $y=b$ so $b^{3}=b$ and we have the following relations:

$$
b=b \cdot b^{2} \cdot b^{2}, b^{2}=b \cdot b \cdot b^{2},
$$

which shows that $b^{2} \in[b]_{\mathcal{J}}$ and so $\left|[b]_{\mathfrak{J}}\right|>1$.

Proof of Proposition A. We consider the different cases as follows:

Case 1. $x \mathcal{L} y \Longrightarrow x \mathcal{R} y$. If $x \mathcal{L} y$ so, $y=x u$ and $x=y v$, for some $u, v \in S$. Since $S$ is quasi-commutative then there exist integers $r_{u}, r_{v}$ such that $x u=u^{r_{u}} x$ and $y v=v^{r_{v}} y$, respectively. Therefore, the identities $y=u_{1} x$ and $x=v_{1} y$ show that $x \mathcal{R} y$, where $u_{1}=u^{r_{u}}$ and $v_{1}=v^{r_{v}}$.

Case 2. $x \mathcal{R} y \Longrightarrow x \mathcal{L} y$. In a similar way to the first case and considering the definition of the right Green graphs.

Case 3. $x \mathcal{L} y \Longleftrightarrow x \mathcal{H} y$. As in Case $1, x \mathcal{L} y$ yields $x \mathcal{R} y$. So, by the definition of $\mathcal{H}$-relation, we get $x \mathcal{H} y$. The converse is obvious.

Case 4. $x \mathcal{R} y \Longleftrightarrow x \mathcal{H} y$. Similar to Cases 2 and 3 .

Case 5. $x \mathcal{L} y \Longrightarrow x \mathcal{J} y$. If $x \mathcal{L} y$ then there exist $u, v \in S$ such that $y=x u$ and $x=y v$. Due to the quasicommutativity of $S$, there exist positive integers $r_{v}, r_{u}, r_{y}, r_{x}$ such that

$$
y v=v^{r_{v}} y, x u=u^{r_{u}} x, v y=y^{r_{y}} v, u x=x^{r_{x}} u \text {. }
$$

There are three cases to consider:

(1) $r_{v}>1, r_{y}>1$. We get:

$$
x=y v=v^{r_{v}} y=v^{r_{v}-1}(v y)=v^{r_{v}-1}\left(y^{r_{y}} v\right)=\left(v^{r_{v}-1}\right) y\left(y^{r_{y}-1} v\right),
$$

which yields $x=u_{1} y v_{1},\left(u_{1}=v^{r_{v}-1}, v_{1}=y^{r_{y}-1} v\right)$.

(2) $r_{v}=1, r_{y} \geqslant 1$. We get:

$$
x=y v=v y=v(x u)=v(y v) u=u_{2} y v_{2},\left(u_{2}=v, v_{2}=v u\right) .
$$

(3) $r_{v}>1, r_{y}=1$. In this situation, we have:

$$
x=y v=v^{r_{v}} y=v^{r_{v}-1}(v y)=v^{r_{v}-1}(y v),
$$

which yields $x=u_{3} y v_{3},\left(u_{3}=v^{r_{v}-1}, v_{3}=v\right)$. The proof of $y=u_{i} x v_{j}$ for some $u_{i}, v_{j} \in S$ is similar.

Case 6. $x \mathcal{R} y \Longrightarrow x \mathcal{J} y$. Clearly, $x \mathcal{R} y$ yields $x \mathcal{L} y$ so, $x \mathcal{J} y$. 
Case 7. $x \mathcal{H} y \Longrightarrow x \mathcal{L} y$. $x \mathcal{J} y$ implies that $x=u_{1} y v_{1}$ and $y=u_{2} x v_{2}$ for some $u_{1}, u_{2}, v_{1}$ and $v_{2}$ in $S$. Because of the quasi-commutativity of $S$, we have $x=\left(y^{r_{y}} u_{1}\right) v_{1}=y u_{2},\left(u_{2}=y^{r_{y}-1} u_{1} v_{1}\right)$ and $y=\left(x^{r_{x}} u_{2}\right) v_{2}=x v_{3}$, $\left(v_{3}=x^{r_{x}-1} u_{2} v_{2}\right)$ where $r_{y}$ and $r_{x}$ are both positive integers. This shows that $x \mathcal{L} y$.

Case 8. $x \mathcal{D} y \Longrightarrow x \mathcal{J y}$. Since $\mathcal{D}$ is the smallest equivalence relation containing $\mathcal{L}$ and $\mathcal{H}$, then $\mathcal{D} \subseteq \mathcal{L}$. So, the proof is obvious.

Case 9. $x \mathcal{J} y \Longrightarrow x \mathcal{D} y$. Let $x \mathcal{J} y$. Then, there are elements $\mathfrak{u}_{1}, \mathfrak{u}_{2}, v_{1}$ and $v_{2}$ in $S$ such that

$$
x=u_{1} y v_{1}, y=u_{2} x v_{2} \text {. }
$$

Setting $z=u_{1} y, k=v_{1}$ yields $x=u_{1} y v_{1}=z k$. So, $z=u_{1} y=u_{1}\left(u_{2} x v_{2}\right)$. By the quasi-commutativity of $S$, there are integers $r_{1}, r_{2}$ such that $u_{2} x=x^{r_{1}} u_{2}, u_{1} x=x^{r_{2}} u_{1}$. Therefore,

$$
z=u_{1}\left(u_{2} x v_{2}\right)=u_{1}\left(x^{r_{1}} u_{2}\right) v_{2}=\left(u_{1} x\right)\left(x^{r_{1}-1} u_{2} v_{2}\right)=x u_{3},
$$

where, $u_{3}=\left(x^{r_{2}-1} u_{1}\right)\left(x^{r_{1}-1} u_{2} v_{2}\right)$. This shows that $x \mathcal{L} z$. Moreover, $y=u_{2} x v_{2}=u_{2}\left(z v_{3}\right)$ where, $v_{3}=v_{1} v_{2}$ and so there is an integer $r_{v_{3}} \geqslant 1$ such that

$$
y=u_{2} x v_{2}=v_{4} z,\left(v_{4}=u_{2} v_{3}^{r_{v_{3}}}\right) \text {. }
$$

The latter identity and $z=\mathfrak{u}_{1} y$ confirm that $z \mathcal{R} y$. This completes the proof of $x \mathcal{D} y$.

Proof of Proposition B. Let $x \mathfrak{J b}$ where $x \in S$ and $x \neq b$. So, there exist elements $u_{i}, v_{i} \in S,(i=1,2)$ such that

$$
\mathrm{b}=\mathrm{u}_{1} x v_{1}, x=\mathrm{u}_{2} \mathrm{~b} v_{2} .
$$

So we have $b=u_{1}\left(u_{2} b v_{2}\right) v_{1}=u_{3} b v_{3}$ where $u_{3}=u_{1} u_{2}, v_{3}=v_{2} v_{1}$. Because of the quasi-commutativity of $S$, we can find positive integers $r_{b}$ such that $u_{3} b=b^{r_{b}} u_{3}$ and therefore $b=b^{r_{b}} y$ where $y=u_{3} v_{3}$. Considering two different cases for $r_{b}$, we have:

(1) If $r_{b}>1$ so $b=b^{r_{b}} y=b^{r_{b}-1}(b y)$ and by quasi-commutativity of $S$ we have $b=b^{r_{b}-1} y^{r_{y}} b$ where $r_{y}$ is some positive integer.

(2) If $r_{b}=1$ then $u_{3} b=b u_{3}$ and so the nowhere commutativity of the semigroup gives $u_{3}=b$.

Therefore by the quasi-commutativity of $S$ we have:

$$
\mathrm{b}=\mathrm{u}_{3} \mathrm{~b} v_{3}=\mathrm{b} \cdot\left(v_{3}^{r_{v_{3}}} \mathrm{~b}\right)=\mathrm{b} \cdot v_{4} \cdot \mathrm{b},\left(v_{4}=v_{3}^{r_{v_{3}}}\right),
$$

where $r_{v_{3}}$ is a positive integer. This means that $b$ is a regular element of $S$. For the converse, we consider Lemma 2.1.

Proof of Proposition C. By using Proposition A, we get that

$$
\Gamma_{\mathcal{L}}(S) \cong \Gamma_{\mathcal{R}}(S) \cong \Gamma_{\mathcal{J}}(S) \cong \Gamma_{\mathcal{D}}(S) \cong \Gamma_{\mathcal{H}}(S) \text {. }
$$

So, identifying the Green graph of $S$ one needs only to consider the $\mathcal{L}$-classes of $S$. If there are $n$ nonregular elements $b_{1}, b_{2}, \cdots, b_{n} \in S$ then by a consequence of Proposition $B$, we get:

$$
n K_{1}=\bigcup_{1}^{n} \Gamma_{\mathcal{L}}\left(\left[b_{i}\right]\right) .
$$

By considering the set of all $\mathcal{L}$-classes of $S$ as $\left\{\mathcal{L}_{1}, \mathcal{L}_{2}, \cdots, \mathcal{L}_{\mathrm{m}}\right\}$, where each class contains at least two elements, we construct the sub-graph $\mathrm{K}_{\mathrm{m}}$ of $\Gamma_{\mathcal{L}}(\mathrm{S})$. Consequently,

$$
\Gamma_{\mathcal{L}}(\mathrm{S}) \cong n K_{1} \cup \mathrm{K}_{\mathrm{m}} \text {. }
$$

Since $S$ is non-regular, $\Gamma_{\mathcal{L}}(S)$ is not a complete graph.

Conclusion 2.2. Using a similar proof, we may extend Proposition A for quasi-hamiltonian semigroups. By definition, the semigroup $S$ is quasi-hamiltonian if and only if for every elements $a, b \in S$ there are positive integers $r_{a}, r_{b}$ such that $a b=b^{r_{b}} a^{r_{a}}$. 


\section{References}

[1] E. Adan-Bante, Conjugacy classes and finite p-groups, Arch. Math. (Basel), 85 (2005), 297-303.1

[2] A. Cherubini, A. Varisco, Quasi-commutative semigroups and o-reflexive semigroups, Semigroup Forum, 19 (1980), 313-321.1

[3] A. H. Clifford, G. B. Preston, The algebraic theory of semigroups I, Amer. Math. Soc., Providence, (1961). 1, 2

[4] A. Gharibkhajeh, H. Dosstie, A graphical difference between the inverse and regular semigroups, Bull. Iranian Math. Soc., 40 (2014), 413-421.1

[5] A. Nagy, Special classes of Semigroups, Kluwer Academic Publishers, Dordrecht, (2001).1

[6] N. P. Mukherjee, Quasi-commutative semigroups I, Czechoslovak Math. J., 22 (1972), 449-453.

[7] M. R. Sorouhesh, H. Dosstie, Quasi-commutative semigroups of finite order related to Hamiltonian groups, Bull. Korean Math. Soc., 52 (2015), 239-246.1 